\title{
Studies of top-quark properties and search for electroweak single top-quark production at the Tevatron
}

\author{
Mousumi Datta $^{\text {a* }}$, on behalf of the CDF and D0 Collaborations. \\ ${ }^{a}$ Fermi National Accelerator Laboratory, \\ P.O. Box 500, Batavia, IL 60510-0500, USA
}

The top quark was discovered in 1995 by the CDF and D0 experiments at the Fermilab Tevatron during the Run I operation. Since the start of the Tevatron Run II in 2001, both experiments have collected $\sim 2 \mathrm{fb}^{-1} \mathrm{data}$ samples, which are over twenty times larger than that used in the Run 1 discovery. This larger data sample allows more precise studies of top-quark properties; differences between observed top-quark properties and the Standard Model (SM) prediction may give hints to possible physics beyond the SM. Here we present the latest results on the measurements of top-quark properties and the search for electroweak (EW) single top quark production from the CDF and D0 collaborations. The integrated luminosity used for the measurements corresponds to about 1 $\mathrm{fb}^{-1}$.

\section{INTRODUCTION}

The existence of the top quark is predicted by the SM. The top quark $\left(T_{3}=+1 / 2\right.$ and $Q=2 / 3)$ is the weak iso-spin partner of the bottom quark. The mass of the top quark $m_{t}$ is about $171 \mathrm{GeV} / \mathrm{c}^{2}[1]$, which is much larger than the masses of all the other quarks and is in the same order of magnitude as the masses of $W$ and $Z$ bosons. The large mass makes top-quark properties unique and interesting both from the viewpoint of the SM and beyond. Due to the large mass the top quark lifetime in the SM is expected to be about $0.5 \times 10^{-24} \mathrm{~s}$. As a result unlike any other quark the top quark in the SM decays before hadronization [2], providing us with the unique opportunity to study the properties of a "bare" quark. Furthermore, the top quark might provide insight to EW-symmetry-breaking mechanism and could be a probe to beyond the SM physics [3].

The top quark was discovered about twelve years ago by the CDF and D0 experiments in

\footnotetext{
*Thanks to my colleagues from CDF and D0 collaborations for providing materials and suggestions for the presentation and this manuscript. I would like to thank Dr. D. A. Glenzinski for his feedbacks while preparing the presentation and manuscript.
}

$p \bar{p}$ collisions at the Fermilab Tevatron. To-date all the direct top quark measurements come from these two experiments. In the hadron collisions top quarks are predominantly produced in pairs through the QCD processes $q \bar{q} \rightarrow t \bar{t}$ and $g g \rightarrow t \bar{t}$. For Tevatron Run II at $\sqrt{s}=1.96 \mathrm{TeV}$ the expected $t \bar{t}$ production cross section is about $7 \mathrm{pb}$ [4] for $m_{t}=175 \mathrm{GeV} / \mathrm{c}^{2}$. Top quark can also be produced in hadron collisions singly though EW processes. The cross sections for single top production in $s$-channel and $t$-channel at $\sqrt{s}=1.96$ $\mathrm{TeV}$ are $0.88 \pm 0.11 \mathrm{pb}$ and $1.98 \pm 0.25 \mathrm{pb}$, respectively [5] for $m_{t}=175 \mathrm{GeV} / \mathrm{c}^{2}$.

In the SM top quark decays to a $W$ boson and a $b$ quark most of the time. The $t \bar{t}$ final states are divided into three classes based on the decay mode of the two $W$ bosons:

1. dilepton: $t \bar{t} \rightarrow W^{+} b W^{-} \bar{b} \rightarrow \bar{\ell} \nu_{\ell} b \ell^{\prime} \bar{\nu}_{\ell^{\prime}} \bar{b}$, branching ratio $\sim 10 \%$, relatively low background;

2. lepton+jets: $t \bar{t} \rightarrow W^{+} b W^{-} \bar{b} \rightarrow q \bar{q}^{\prime} b \ell \bar{\nu}_{\ell} \bar{b}+$ $\bar{\ell} \nu_{\ell} b q \bar{q}^{\prime} \bar{b}$, branching ratio $\sim 43 \%$, medium background level; and

3. all hadronic: $t \bar{t} \rightarrow W^{+} b W^{-} \bar{b} \rightarrow q \bar{q}^{\prime} b q^{\prime \prime} \bar{q}^{\prime \prime \prime} \bar{b}$, branching ratio $\sim 46 \%$, suffers from large QCD background. 
Here $\ell=e, \mu$, or $\tau$, though most of the lepton + jets and dilepton analyses rely only on the $e$ and $\mu$ decay modes.

The CDF and D0 experiments have studied various aspects of the top quark [6,7]. In this proceeding we present some of the recent results on top quark physics using $\sim 1 \mathrm{fb}^{-1} p \bar{p}$ collision data at $\sqrt{s}=1.96 \mathrm{TeV}$ collected between March 2002 and March 2006. The topics are divided into several categories as follows:

- $t \bar{t}$ production: $t \bar{t}$ production cross section, and $t \bar{t}$ production mechanisms;

- Decay properties: helicity of the W-boson from top decay;

- Characteristics : top quark charge;

- EW single top production; and

- Search for beyond the SM physics.

\section{Measurement of $t \bar{t}$ Production Cross Section}

The CDF and D0 experiments have measured the $t \bar{t}$ production cross section in different decay channels and using various methods. These measurements are sensitive to different systematic effects and provide tests of Quantum Cromo Dynamics (QCD) calculations.

A brief description of the most precise $t \bar{t}$ cross section measurements to-date are as follows. The analyses are performed by the CDF and D0 collaborations in the lepton+jets channel using btagged events $[8,9]$. The CDF analysis uses secondary vertex b-tagging algorithm [10], which has an efficiency of $\sim 40 \%$ per b-jet with a misidentification rate of $\sim 0.5 \%$. The events are selected requiring a lepton ( $e$ or $\mu$ ) with transverse momentum $P_{T}>20 \mathrm{GeV} / \mathrm{c}$, a missing transverse energy $M E T>30 \mathrm{GeV}$, and three or more jets with transverse energy $E_{T}>20$ $\mathrm{GeV}$ and at least one of the jets being identified as a b-jet. Figure 1 shows the jet multiplicity distributions for CDF II data, $t \bar{t}$ and background events. Events with three or more jets, which are used for the CDF measurement, have a $t \bar{t}$ content of $80 \%$ and higher. The measured $t \bar{t}$ cross section by the CDF collaboration is: $8.2 \pm 0.5$ (stat) \pm 0.8 (syst) \pm 0.5 (lum) pb. The D0 analysis selects events with a high $P_{T}$ lepton, large $M E T$, three or more high $E_{T}$ jets and at least one of the the jets being identified as a b-jet using a lifetime b-tagging algorithm [9]. The $t \bar{t}$ cross section measured by the D0 collaboration is: $8.3_{-0.5}^{+0.6}(\text { stat })_{-1.0}^{+0.9}($ syst $) \pm 0.5($ lum $)$ pb. Both measurements are limited by systematic uncertainties, where the major sources are luminosity $(\sim 6 \%)$, b-tagging $(\sim 6 \%)$, parton density functions, and modeling of signal and background.

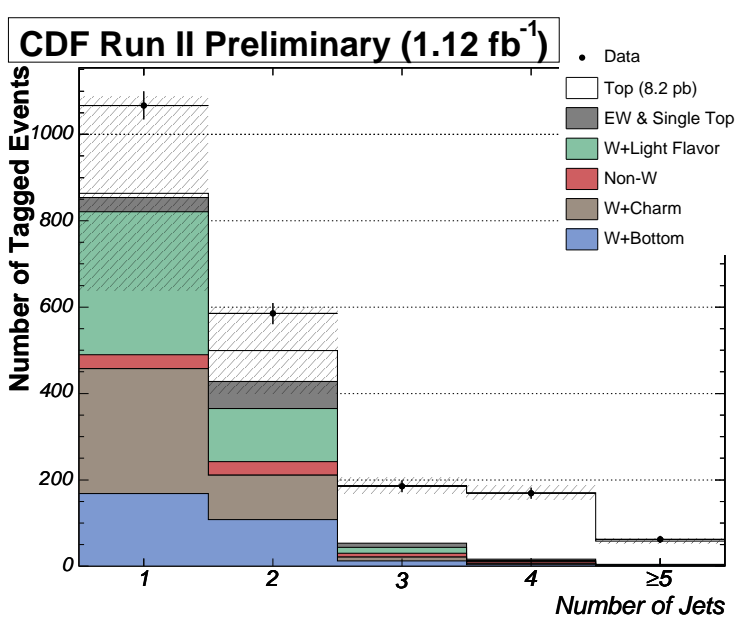

Figure 1. Distribution of jet multiplicity for events with at least one b-tagged jet. The $t \bar{t}$ contribution is normalized to the measured cross section value.

A summery of the recent cross section measurements is shown in Figure 2 [6,7]; which shows that all the measurements are consistent with each other and are in agreement with the theoretical expectations. 


\section{3. $t \bar{t}$ Production Mechanism}

At Tevatron the SM prediction for $q \bar{q} \rightarrow t \bar{t}$ and $g g \rightarrow t \bar{t}$ are $\sim 85 \%$ and $\sim 15 \%$, respectively. However these predictions have larger uncertainties [4]. The measurement of the relative fraction of these processes provide a test of perturbative QCD calculation, and is sensitive to new $t \bar{t}$ production mechanisms. The CDF collaboration has measured the fraction $\sigma(g g \rightarrow t \bar{t}) / \sigma(p \bar{p} \rightarrow t \bar{t})$, using two different analysis methods, discussed as follows. Both analyses use lepton+jets decay channel and select events with $W(\ell \nu)$ and four or more jets with at least one b-tagged jet.

One analysis [11] takes advantage of the fact the $g g$ production channel has larger number of low energy particles compared to the $q \bar{q}$ production channel. Data and Monte Carlo samples for dijet and $\mathrm{W}+$ jets processes are used to demonstrate the correlation between average number of gluons $\left\langle N_{g}>\right.$ and average number of low $P_{T}$ tracks $<N_{t r k}>$ in the events. The $<N_{t r k}>$ distributions for " $q \bar{q}$ " and " $g g$ " initial state processes are obtained using $W+0$ jets data and dijet data, respectively, which are used as templates to reconstruct likelihood function. From the likelihood fit to the $t \bar{t}$ enriched data events and after taking into account of the background contributions we get $\sigma(g g \rightarrow t \bar{t}) / \sigma(p \bar{p} \rightarrow t \bar{t})=$ $0.01 \pm 0.16$ (stat) \pm 0.07 (syst).

The other analysis [12] discriminates between $q \bar{q} \rightarrow t \bar{t}$ and $g g \rightarrow t \bar{t}$ processes using production and decay kinematics. After reconstructing the kinematics of $t \bar{t}$ event, eight kinematic variables are calculated: two variables describing the production of the event; the remaining six variables describing the decay using information about spin correlations. The variables are used as inputs to a Artificial Neural Network (NN), which is trained to distinguish between $q \bar{q} \rightarrow t \bar{t}$ and $g g \rightarrow t \bar{t}$ processes. The NN output shapes are used as templates to construct a likelihood. The obtained constrains on the $\sigma(g g \rightarrow t \bar{t}) / \sigma(p \bar{p} \rightarrow t \bar{t})$ are $<0.33$ at $68 \%$ confidence level (CL), and $<0.61$ at $95 \%$ CL.

\section{Helicity of the W Boson from Top Decay}

In the SM the fractions for longitudinally $f_{0}$, left-handed $f_{-}$and right-handed $f_{+}$W bosons from top decays are predicted to be $0.7,0.3$ and 0.0 , respectively [13]. The SM expectation might be modified due to beyond SM contributions [13, 14].

Both the CDF and D0 collaborations have performed measurements of the $W$-boson helicity fractions [15]. Here we discuss the recent measurements at the CDF experiment. The variable used for measuring the $\mathrm{W}$-boson helicity fractions is $\cos \theta^{*}$, which is the angle between the lepton and the $\mathrm{W}$ boson in the top rest frame. Due to the helicity conservation $d \sigma / d \cos \theta^{*}$ is proportional to $\left(1-\cos ^{2} \theta^{*}\right),\left(1-\cos \theta^{*}\right)^{2}$ and $\left(1+\cos \theta^{*}\right)^{2}$, for longitudinal, left-handed and right-handed $\mathrm{W}$ bosons, respectively.

Two similar analyses are performed in lepton+jets decay channel, using events containing $W(\ell \nu)$, and four or more jets with at least one identified b-jet. The $\cos \theta^{*}$ variable is obtained from the kinematics of the reconstructed $t \bar{t}$ events. The reconstructed $\cos \theta^{*}$ distributions for longitudinal, left-handed and right-handed Wbosons are used as temples in a likelihood fit to measure $f_{0}$ and $f_{+}$.

Both analyses obtain very similar results; the measurement from one of the analyses are quoted here. From simultaneous measurement of $f_{0}$ and $f_{+}$we get: $f_{0}=0.74 \pm 0.25$ (stat) \pm 0.06 (syst) and $f_{+}=-0.06 \pm 0.10$ (stat) \pm 0.03 (syst). In addition, $f_{0}\left(f_{+}\right)$is measured by keeping $f_{+}\left(f_{0}\right)$ fixed to the SM expected values. The results are: $f_{0}=0.61 \pm 0.12$ (stat) \pm 0.06 (syst) with $f_{+}=0.0$; and $f_{+}<0.11$ at $95 \% \mathrm{CL}$ with $f_{0}=0.7$. The measurements are consistent with the SM expectation. However, currently the analyses are limited by statistics.

\section{Top Quark Charge}

The SM predicts the charge of the top quark to be $+2 e / 3$. In order to confirm that the observed particle with mass $\sim 171 \mathrm{GeV} / \mathrm{c}^{2}$ is the SM top quark we measure its charge. Most of the measurements using $t \bar{t}$ events pair a $W$ with 
a $b$-quark. For $W \rightarrow \ell \nu$, the charge of the $\mathrm{W}$ is known from the charge of the lepton. However, we do not distinguish between a $b$-quark and $\bar{b}$ quark. Therefore, the charge of the $W b$ pair can be $+2 e / 3$ or $-4 e / 3$. It has been postulated [16] that the observed quark with mass $\sim 171 \mathrm{GeV} / \mathrm{c}^{2}$ has a charge of $+4 e / 3$ and is not the SM top quark.

Both CDF and D0 experiments perform measurements $[17,18]$ to distinguish between $+2 e / 3$ and $-4 e / 3$ scenarios. Here we discuss the CDF analysis [17]. The top charge is measured in lepton+jets and dilepton channels. There are three main components to this measurement: (1) the charge of the $W$ : determined using the charge of the lepton(s); (2) the flavor of the bjet: obtained using weighted jet charge algorithm: $Q_{\mathrm{b}-\mathrm{jet}}=\left(\sum_{i} q_{i} \cdot\left(\vec{p}_{i} \cdot \hat{a}\right)^{0.5}\right) /\left(\sum_{i}\left(\vec{p}_{i} \cdot \hat{a}\right)^{0.5}\right)$, where $q_{i}$ and $\vec{p}_{i}$ are the charge and momentum of the tracks within the jet, and $\hat{a}$ is the jet axis; and (3) Pairing the $\mathrm{W}$ with the $\mathrm{b}$ jet to ensure $\mathrm{W}$ and the $\mathrm{b}$ jet come from the same top decay branch.

In the CDF data 62 pairs with charge $+2 e / 3$ and 48 pairs with charge $-4 e / 3$ have been observed. The result is obtained using a profile likelihood which uses observed number of data events and incorporates the purities for jet-charge algorithm and $W b$ pairing. We find that the charge is consistent with the $+2 e / 3$ hypothesis. The analysis exclude charge $-4 e / 3$ scenario with $81 \%$ confidence, where the probability of incorrectly rejecting the SM is a priori defined to be $1 \%$.

\section{EW Single Top Production}

The single top production is an important and interesting process. In the SM context it provides the only way to directly measure the CKM matrix element $\left|V_{t b}\right|$, and can be used to test the V-A structure of the top charge current interaction. The single top rate is also sensitive to beyond SM physics [19]. The SM expectation of single top production in s- and t-channel combined is about $2.9 \mathrm{pb}$ [5].

Here recent $\mathrm{CDF}$ and D0 searches for single top production are discussed [20-24]. The major obstacle in finding single top is the large background. The leptonic $W$ decay channel is used in order to reduce multi-jet backgrounds. Events are required to have high-energy $e$ or $\mu$ and large $M E T$. The CDF analyses use events with exactly two high energy jets, which is the most sensitive search region. The D0 analyses use events with two, three and four jets; events are analyzed in different subsets to take advantage of relative signal-to-background fractions (S/B). Both CDF and D0 analyses require at least one of the jets to be an identified b-jet, which is crucial for significantly improving S/B. The signal and background expectations and data yields are listed in Table 1. In the $\mathrm{W}+2$ jets sample the $\mathrm{S} / \mathrm{B}$ are $\sim 1 / 15$ and $\sim 1 / 19$, respectively, for $\mathrm{CDF}$ and D0 experiments.

In order to extract single top signal from the challenging background dominated dataset both experiments use various multi variate techniques. The CDF experiment uses NN [20], likelihood discriminant [21] and matrix element (ME) discriminant [22]. The D0 experiment also applies thee methods [23]: boosted decision tree (DT), ME discriminant and Bayesian NN.

The expected sensitivity and observed result from each method and combined D0 measurement [24] are listed in Table 2. The D0 decision tree measurement has a significance of $3.4 \sigma$, and is the first evidence of single top production.

\section{Search for Beyond the SM processes}

Many beyond SM theories predicts resonant $t \bar{t}$ production from massive $Z^{\prime}$ boson [25] decay and resonant $t \bar{b}$ production from massive $W^{\prime}$ boson [26] decay. The $t \bar{t}$ and single top channels are used to search for $Z^{\prime}$ and $W^{\prime}$, respectively $[27,28]$. Here the recent measurements from the CDF experiment are mentioned. Resonant $t \bar{t}$ and $t \bar{b}$ productions are modeled by heavy neutral and charged bosons, respectively, with SM-like coupling to fermions. Analyses assume a narrow width for the resonances.

The $t \bar{t}$ and $t \bar{b}$ invariant mass spectra are studied and limits on resonant production mechanism are set as a function of the corresponding resonance mass. The limits are used for constraining some of the theory models. For example, from $t \bar{t}$ resonance searches a leptophobic $Z^{\prime}$ predicted 
Table 1

Expected single top signal and background and observed number of data events.

\begin{tabular}{l|l|lll}
\hline & at CDF & \multicolumn{3}{|c}{ at D0 } \\
& $W(\ell \nu)+2$ jets & $W(\ell \nu)+2$ jets & $W(\ell \nu)+3$ jets & $W(\ell \nu)+4$ jets \\
\hline Single top: s-channel & $15 \pm 2$ & $16 \pm 3$ & $8 \pm 2$ & $2 \pm 1$ \\
\hline Single top: t-channel & $22 \pm 4$ & $20 \pm 4$ & $12 \pm 3$ & $4 \pm 1$ \\
\hline Total background & $549 \pm 95$ & $686 \pm 41$ & $460 \pm 39$ & $253 \pm 38$ \\
\hline Observed & 644 & 697 & 455 & 246 \\
\hline
\end{tabular}

Table 2

Results and expected sensitivity from various multi variate analyses used for single top searches.

\begin{tabular}{llll}
\hline Multi variate Methods & $\begin{array}{l}\text { Expected Significance } \\
\text { at the SM rate }\end{array}$ & $\begin{array}{l}\text { Measured Cross section } \\
\text { Combined s- and t- channel } \\
(\mathrm{pb})\end{array}$ & $\begin{array}{l}\text { Significance of the } \\
\text { Measurement }\end{array}$ \\
\hline NN (CDF) & $2.6 \sigma$ & $0.0_{-0.0}^{+1.2}(<2.6$ at 95\% C.L. $)$ & - \\
\hline Likelihood (CDF) & $2.0 \sigma$ & $0.3_{-0.3}^{+1.2}(<2.7$ at 95\% C.L. $)$ & - \\
\hline Matrix Element (CDF) & $2.5 \sigma$ & $2.7_{-1.3}^{+1.5}$ & $2.3 \sigma$ \\
\hline Decision Tree (D0) & $2.1 \sigma$ & $4.9 \pm 1.4$ & $3.4 \sigma$ \\
\hline Matrix Element (D0) & $1.8 \sigma$ & $4.6_{-1.5}^{+1.8}$ & $2.9 \sigma$ \\
\hline Bayesian NN (D0) & $1.3 \sigma$ & $5.0 \pm 1.9$ & $2.4 \sigma$ \\
\hline D0 Combined & - & $4.8 \pm 1.3$ & $3.5 \sigma$ \\
\hline
\end{tabular}

by the topcolor theory [25] is ruled out below 720 $\mathrm{GeV} / \mathrm{c}^{2}$ at $95 \% \mathrm{CL}$. The $t \bar{b}$ resonance search excludes $W^{\prime}$ mass $m_{W^{\prime}}$ less than 760 (790) $\mathrm{GeV} / \mathrm{c}^{2}$ for $m_{W^{\prime}}>m_{\nu_{R}}\left(m_{W^{\prime}}<m_{\nu_{R}}\right)$ [26] at 95\% CL.

\section{Conclusion}

The the recent results from the measurements for top-quark properties at the Tevatron are presented. Many interesting measurements with the $t \bar{t}$ sample, search for single top production and beyond SM physics searches with the top samples are discussed here. Besides some of the cross section measurements most of the measurements and searches are limited by statistics. Even though the Tevatron has about twenty times larger $t \bar{t}$ sample compared to that in Run I, the current samples consists of few hundred signal event. Most of the analysis will be updated with about $2 \mathrm{fb}^{-1}$ data by the end of this year. With the increasing dataset $\left(6-8 \mathrm{fb}^{-1}\right.$ by 2010$)$ the true na- ture of the top quark will be further unveiled.

\section{REFERENCES}

1. CDF Collaboration and D0 Collaboration, FERMILAB-TM-2380-E, TEVEWWGTOP-2007-01, CDF-NOTE-8735, D0-NOTE5378 (2007).

2. Physics Letter B 181, 157 (1986).

3. T. Tait, in these proceedings.

4. Journal of High Energy Physics 0404, 068 (2004); Physical Review D 68, 114014 (2003).

5. Physical Review D 70, 114012 (2004).

6. http://www-cdf.fnal.gov /physics/new/top/top.html

7. http://www-d0.fnal.gov /Run2Physics/WWW/results/top.htm

8. CDF Collaboration, CDF Public Note 8795 (2007).

9. D0 Collaboration, D0 Conference Note 5355 (2007). 
10. Physical Review D 64, 032002 (2001).

11. CDF Collaboration, CDF Public Note 8724 (2007).

12. CDF Collaboration, CDF Public Note 8725 (2007).

13. Physical Review D 45, 124 (1992).

14. Physical Review Letters 38, 1252 (1977); Journal of Physics G 26, 99 (2000); Physical Review D 65, 053002 (2002).

15. CDF Collaboration, CDF Public Note 8380 (2006); CDF Collaboration, CDF Public Note 8368 (2006); Physical Review Letters 98, 072001 (2007); Physical Review D 75, 052001 (2007); Physical Review D 75, 031102 (2007).

16. Physical Review D 59, 091503 (1999).

17. CDF Collaboration, CDF Public Note 8782 (2007).

18. Physical Review Letters 98, 041801 (2007).

19. Physical Review D 63, 014018 (2001).

20. CDF Collaboration, CDF Public Note 8677 (2007).

21. CDF Collaboration, CDF Public Note 8585 (2006).

22. CDF Collaboration, CDF Public Note 8588 (2006).

23. Physical Review Letters 98, 181802 (2007).

24. D0 Collaboration, D0 Conference Note 5342 (2007).

25. Physics Report 317, 143 (1999); CERNTH/96-169 (1996); B. Lillie et. al., (hepph/0701166); Physical Review D 61, 055005 (2000); Physics Letter B 200, 211 (1988); Physical Review D 49, 4454 (1994).

26. Physical Review D 10, 275 (1974); Physical Review D 11, 566 (1975); Physics Letters B 385,304 (1996).

27. CDF Collaboration, CDF Public Note 8675 (2007).

28. CDF Collaboration, CDF Public Note 8747 (2007); Physics Letters B 641, 423 (2006).
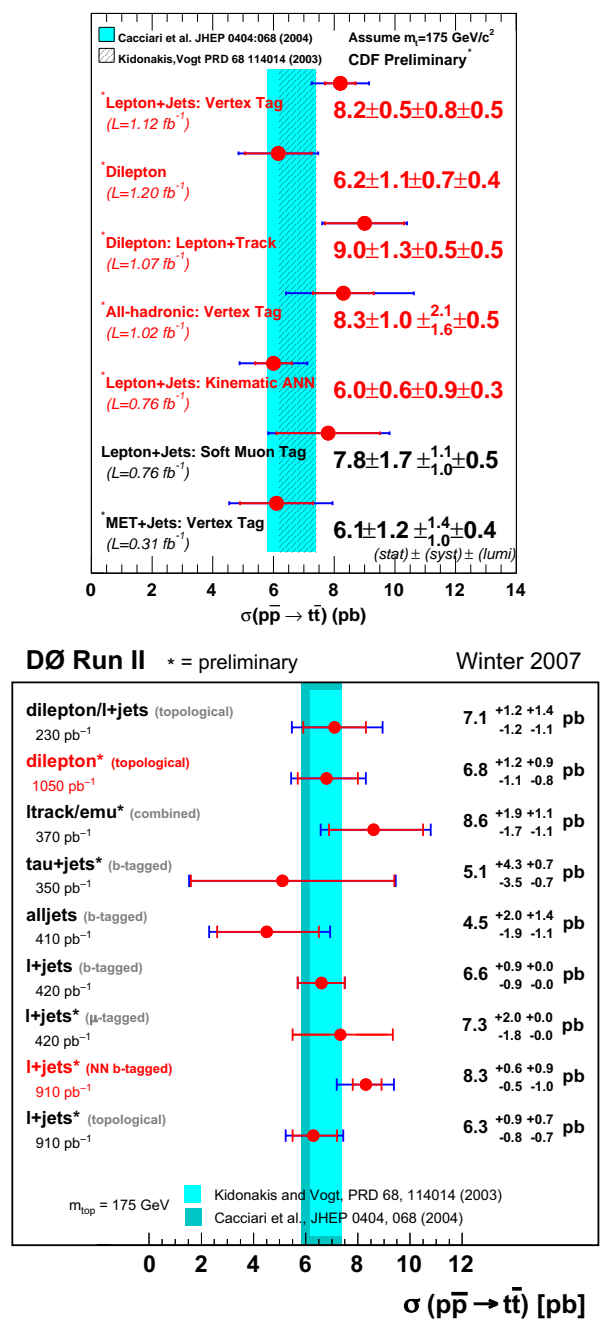

Figure 2. Measurements of the $t \bar{t}$ production cross section at $\sqrt{s}=1.96 \mathrm{TeV}$ for $m_{t}=175$ $\mathrm{GeV} / \mathrm{c}^{2}$ performed by the CDF (top) and D0 (bottom) collaborations. 